\title{
A Comparative Performance Analysis of AODV, DSR and DSDV Protocol for Mobile Ad-hoc Networks
}

\author{
Sandeep Sharma ${ }^{1}$, Pankaj Jagtap ${ }^{2}$ \\ M.Tech (Network Management \& Information Security), School of Computer Science and IT, Devi Ahilya University, \\ Indore, India ${ }^{1}$
}

Lecturer, School of Computer Science and IT, Devi Ahilya University, Indore, India ${ }^{2}$

\begin{abstract}
The quick growth of mobile communication in current years is especially observed in the field of mobile system, wireless local area network, and ubiquitous computing. In this vast of time, ad-hoc networks are extremely active networks characterized by the lack of physical infrastructure. Hubs of this system's capacity as switch which find and keeps up the route to different hubs in the system. Therefore, for ad-hoc network, routing protocol plays a significant task for building an efficient network. The plan of efficient routing protocols is a basic problem in a Mobile Ad-Hoc Network (MANET). Lots of different protocols have been proposed in the literature, each one based on dissimilar characteristics and properties. In this paper, an attempt has been complete to compare three well known routing protocols Ad-hoc on demand distance vector (AODV), Dynamic Source Routing (DSR) and Destination Sequenced Distance Vector (DSDV) by using three presentation metrics packet delivery ratio (PDR), Routing Overhead and Remain Energy by using the NS-2.34 simulator.
\end{abstract}

Keywords: AODV, DSR, DSDV, NS2, MANET, Routing Protocol.

\section{INTRODUCTION}

An ad hoc system is an arrangement of remote mobile hosts form a temporary network without the assistance of any remains stand-alone framework or centralized administration [1]. Mobile Ad-hoc systems are self-configuring out and self-arranging multi-jump wireless systems where, the structure of the system modifies progressively. This is generally because of the mobility of the hubs [3]. Hubs in these systems exploit the comparative random access to wireless channel, helping in a friendly manner to attractive them in multi-jump forwarding. The hub in the system goes about as hosts as well as switches that route information to/from additional hubs in system [2]. Each device in a MANET is allowed to move independently toward any path and will therefore change it connects to different device frequently. Each must forward traffic unrelated to its have utilizations, as be a switch. Routing in ad hoc systems has been demanding task as far back as the wireless systems came into survival. The primary reason behind this is the steady change in system topology since of high level of hub mobility. A digit of protocols have been produced for finish this task.

\section{A. About MANET}

An ad hoc system is a gathering of hubs that don't require depending on a predefined infrastructure to keep the system associated. Ad hoc network can be figure out, compound together or partitioned into disconnect network on the fly, with no fundamentally depending on a settled framework to run the operation. Hubs of ad hoc network are frequently mobile, which also associates that they relate wireless communication to save the accessibility, in which case the systems are called as mobile ad hoc systems (MANET). Mobility is not, however, a commitment for hubs in ad hoc network $[4,5]$.

A Mobile Ad hoc network (MANET) is an arrangement of remote portable hubs that powerfully self-sort out in discretionary and brief system topologies. Individuals and vehicles can subsequently be internet worked in regions without a previous correspondence foundation or when the utilization of such framework requires remote augmentation. In the versatile specially appointed system, hubs can straightforwardly speak with the various hubs inside their radio reaches; while hubs that not in the immediate correspondence go utilize halfway hub (s) to speak with each other

Figure 1 is the depiction of example of mobile ad hoc structure: 


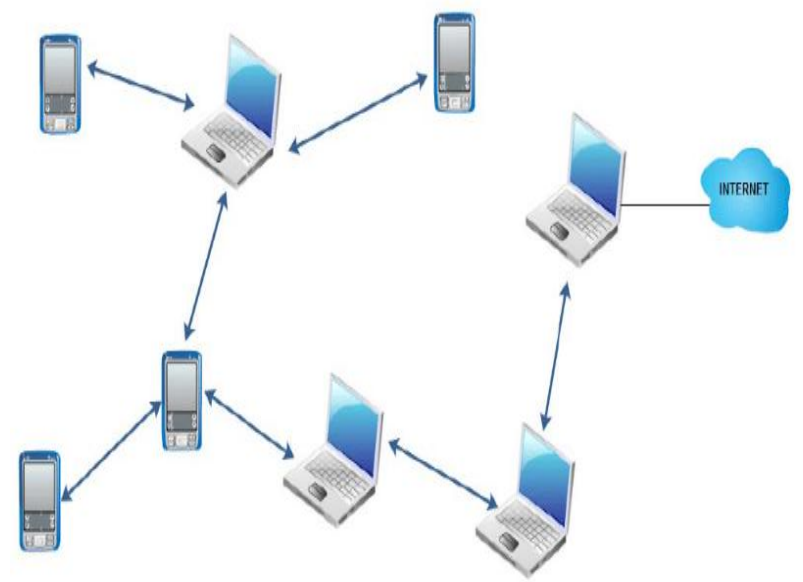

Figure 1: Example of MANET

\section{B. Routing Protocols}

Routing is the act of moving information from a source to a destination in an internetwork. During this process, at least one intermediate node within the internetwork is encountered. Traditional wireless communication networks require a fixed infrastructure over which communication takes place. Therefore, considerable resources and effort are required to set up such networks, even before they can actually be used. Routing is the process of choosing route in a network along which to send network traffic [6]. In packet switching network, routing direct packet sending, transit of logically addressed to packets from their source toward their definitive destination through middle of the road hubs. An ad hoc routing protocol is a gathering, or standard, that controls how hubs choose which approach to route packets between computing devices in a wireless ad hoc network. Routing protocol is usually the set of rules which decides how a message would attain from source to destination. In Ad-hoc organize, the routing protocols decide and search the route between different nodes. However, these routing protocols are hard to design in those systems where topology varies significantly. So, these protocols need to perform in dynamic environment in an competent way with limited resources [7].

In order to facilitate communication inside the system, a routing protocol is utilized to find route between nodes. The essential objective of such a ad hoc network routing protocol is right and effective route foundation between a pair of hubs with the destination that messages might be conveyed in an timely manner. Route development should to be finished with minimum overhead and bandwidth utilization. An Ad-hoc routing protocol is a convention or standard that controls how hubs come to agree which way to route packets between computing devices in a MANET [8].

Numerous routing protocols have been proposed for the mobile ad hoc network and categorized as Proactive or Table Driven routing Protocol, Reactive or On Demand Routing Protocol.

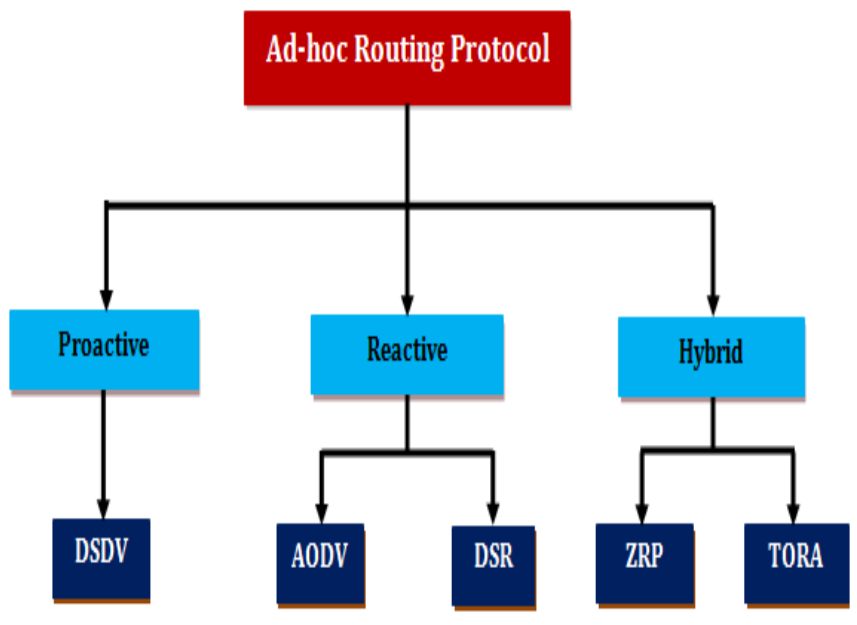

Figure 2: Categorization of Ad-Hoc Routing Protocols

i. $\quad$ Proactive or table-driven routing protocols:

In proactive protocol, every hub keeps up individual steering table containing directing data for each hub in the system. Every hub keeps up steady and current mode steering data by sending control messages occasionally between the hubs 
which refresh their directing tables. The proactive steering conventions utilize connect state directing calculations which much of the time surge the connection data about its neighbors. The downside of proactive directing convention is that every one of the hubs in the system dependably keeps up a refreshed table. Some of the existing proactive routing protocols are DSDV [9] and OLSR [10].

\section{ii. Reactive or On Demand Routing Protocol:}

In Reactive routing protocols, when a source wants to send packets to a destination, it invokes the route discovery mechanisms to find the route to the destination. The route remains legal till the destination is accessible or until the route is no longer desirable. Dissimilar table driven protocols, all nodes need not preserve up-to-date routing information. Some of the most used on demand routing protocols are DSR [10] and AODV [9].

\section{iii. Hybrid Routing Protocol:}

Hybrid routing protocol combines the advantages of both proactive and reactive routing protocols. The routing at first known with numerous proactively prospected routes and then provide the demand from additionally activated nodes throughout reactive flooding. Some of the existing hybrid protocols are ZRP [10] and TORA [11].

The remains of the paper are organized as follows: Section II presents the preceding study for protocol performance. Section III provides an outline and general comparison of the routing protocols used in the study. The simulation environment and presentation results are described in part IV. Finally Section V concludes the paper with future extension of work.

\section{LITERATURE SURVEY}

A large number of papers try to simulate or analyze reactive and proactive protocols in general. The problem of analytical approaches lies in the fact that protocols like DSR, DSDV and AODV are complex and can be configured in many ways to achieve high performance in various scenarios. In this section we list the prior work done in of protocol comparison in ad-hoc environment.

Although several routing protocols have been proposed that can be used in mobile ad hoc networks, there is very little formal analysis that considers the communication overhead or in other word routing overhead for these procedures. Mohammad Naserian et al. [12] provides a new mathematical framework for quantifying the overhead of reactive routing protocols such as Dynamic Source Routing (DSR) and Ad hoc On-Demand Distance Vector Routing (AODV) in wireless ad hoc networks with random location of the nodes. The analysis is compared with the simulations and found to match with the simulation results. The result of this study can be used to predict scalability properties of routing protocols.

Mobile Ad hoc system is system where hubs impart with no focal organization or system framework. They are associated by means of remote channels and can utilize different bounces to trade information. Directing conventions are required for correspondence in such Ad hoc arranges where it focuses for proficient and opportune conveyance of message. There are different execution measurements to think about Ad hoc directing conventions. In this paper Amith Khandakar et al. [13] show a well ordered method is expressed to analyze 3 famous routing conventions, DSR, AODV and DSDV in view of execution measurements Packet Delivery Fraction (Pdf), End to end postpone and Normalized Routing load while changing the quantity of hubs, speed and Pause time. It additionally gives a well ordered approach in view of presumption on the best way to do such a similar review, which could be utilized for future research.

Dilpreet Kaur et al. [14] clarify the characteristics of uniquely routing protocol convention Ad-hoc On Demand Distance Vector Routing (AODV), Optimized interface State Routing (OLSR), Temporally Ordered Routing Algorithm (TORA), Dynamic Source Routing (DSR), Destination-Sequenced Distance-Vector Routing (DSDV) in perspective of the execution estimations like packet movement part, Average delay, Normalized Routing load, Throughput and Jitter under low mobility and low action arrange and in addition under high mobility and high activity organize. Comes about demonstrate that AODV has most outrageous throughput under low action and DSDV has most prominent throughput under high development. As framework winds up perceptibly thick OLSR, DSR and DSDV perform well similar to Throughput than AODV and TORA. TORA performs well in thick frameworks with respect to package transport partition yet meanwhile Normalized Routing pile of TORA is most noteworthy among each one of the traditions in both the frameworks.

Mobile Ad hoc Network (MANET) is a gathering of remote portable hubs that progressively form a system incidentally with no support of focal administration. Besides, every hub in MANET moves subjectively making the multi-jump arrange topology to change arbitrarily at questionable circumstances. There are a few commonplace steering conventions like AODV, DSR, and DSDV and so on which have been proposed for giving correspondence among 
every one of the hubs in the remote system. V. Rajeshkumar et al. [15] exhibit the performance comparison and literature of reactive and proactive protocols AODV, DSR and DSDV based on metrics for example throughput, control overhead, packet delivery ratio and average end-to-end delay by using the NS-2 simulator.

Greed computing has as of late moved from traditional high performance and distributed computing to pervasive and utility figuring in view of the propelled capacities of the remote systems and the lightweight, thin gadgets. This has as result the rise of another registering worldview which is the Mobile Grid. Nor Surayati Mohamad Usop et al. [16] presents the reenactment brings about request to pick the best steering convention to give the most astounding execution when actualize the directing conventions in the objective portable lattice application. The recreations looking at three specially appointed steering conventions named DSDV, DSR and AODV. The reproductions have demonstrated that the regular steering conventions like DSR have a sensational lessening in execution when versatility is high. However the AODV and DSDV are perform extremely well when versatility is high.

\section{COMPARATIVE STUDY}

There are diverse criterion for designing and classifying routing protocols for wireless ad hoc networks. For instance, what routing information is replaced; when and how the routing information is exchanged, when and how routes are computed etc?

\section{A. Discussion}

\section{i. Ad-hoc On Demand Distance Vector (AODV)}

AODV is fundamentally a combination of both DSR and DSDV. It makes use of utilization of the essential on-request system of Route Discovery and Route Maintenance from DSR, in addition to the utilization of jump by-bounce routing, arrangement numbers, and intermittent guides from DSDV. Keeping in mind the end destination to look after courses, AODV typically requires that every hub periodically transmit a HELLO message, with a default rate of once every second. Inability to get three back to back HELLO messages from a neighbor is taken as a sign that the connection to the neighbor in inquiry is down. On the other hand, the AODV determination quickly recommends that a hub may utilize physical layer or connection layer techniques to recognize interface breakages to hubs that it considers neighbors [17] [18].

\section{ii. Destination-Sequenced Distance-Vectors Routing (DSDV)}

DSDV is a table-driven directing component uncommonly delegated convenient frameworks in light of the BellmanFord technique. The standard commitment of the calculation was to deal with the Routing Loop issue which is accessible in Bellman-Ford computation. To do in that capacity, DSDV makes use of plan numbers. Each area in the controlling table contains a series number; the gathering numbers are generally paying little heed to the likelihood that a connection is accessible; else, an odd number is used. The number is created by the goal, and the producer needs to pass on the following refresh with this number. Steering information is appropriated between center points by sending full dumps incidentally and more diminutive incremental updates more generally [19]

\section{iii. Dynamic Source Routing (DSR)}

Dynamic Source Routing (DSR) is a routing protocol for wireless work arranges and depends on a strategy known as source directing. It is like AODV in that it frames a course on-request when a transmitting PC asks for one. But that each middle hub that communicates a course asks for bundle adds its own particular deliver identifier to a rundown conveyed in the parcel. The goal hub produces a course answer message that incorporates the rundown of locations gotten in the course ask for and transmits it back along this way to the source. Route upkeep in DSR is proficient through the affirmations that hubs create when they can confirm that the following hub effectively got a bundle. These affirmations can be connection layer affirmations, uninvolved affirmations or system layer affirmations determined by the DSR convention. Be that as it may, it utilizes source steering as opposed to depending on the directing table at each transitional gadget. At the point when a hub is not ready to confirm the effective gathering of a parcel it tries to retransmit it. At the point when a limited number of retransmissions come up short, the hub creates course mistake message that indicates the dangerous connection, transmitting it to the source hub [18] [20].

\section{B. Comparison of On-Demand and Table Driven Routing Protocols in MANET}

There are three routing protocol which is demonstrate to clarify their applicability. Flat routing protocol distributes routing information to routers that are linked to each other without any association or segmentation arrangement among them. The 3 protocols are act as loop free routing Like that, quick route access, and reduce the packet loss and improve the efficiency of route reconfiguration and less delay (see in table 1). 
Table 1: Protocol Comparison by their working Strategy

\begin{tabular}{|c|c|c|c|}
\hline Parameter & AODV & DSR & DSDV \\
\hline Routing structure & Flat & Flat & Flat \\
\hline Route Acquisition Delay & High & High & Low \\
\hline Control Overhead & Low & Medium & High \\
\hline Loop free & Yes & Yes & Yes \\
\hline Updates Transmitted to & Source & Source & Neighbors \\
\hline
\end{tabular}

\section{SIMULATION AND PERFORMANCE COMPARISON}

\section{A. Simulation}

Simulation is "the procedure of designing a model of a authentic system and behavior experiments with this model for the reason of thoughtful the actions of the system and/or estimating various strategies for the operation of the system". With the dynamic nature of computer networks, we thus actually deal with a dynamic model of a real dynamic system. Network Simulator (NS2.34) is a discrete event simulator directed at networking research.

This section provides the performance analysis of the studied routing protocols. On the basis of the performance comparison an efficient routing protocol is selected which support the small as well as large network efficiently with less resource consumption. Therefore a simulation using NS2 simulator is presented and with the increasing traffic in network the performance is computed using their trace files. The results are report the performance of all the implemented routing protocols for analysis.

A simulator model of a real-world system is essentially a popularization of the real-world system self. In our simulation, 100 mobile nodes move in a 1000 meter x 1000 meter square region for different varying simulation time. All nodes have the same transmission range of 500 meters. The simulation settings and parameters are summarized in table 2. In this work we perform simulation using different number of nodes a 20, 40, 60, 80 and 100.

Table 2: Simulation Parameter

\begin{tabular}{|c|c|}
\hline Parameters & Values \\
\hline Antenna Model & Omni Antenna \\
\hline Simulation Area & $750 X 550$ \\
\hline Radio-Propagation & Two Ray Ground \\
\hline Channel Type & Wireless Channel \\
\hline Traffic Model & CBR \\
\hline Routing Protocol & AODV, DSR, DSDV \\
\hline Mobility Model & Random Waypoint \\
\hline Number of Nodes & $20,40,60,80,100$ \\
\hline
\end{tabular}

B. Performance Comparison
i. Packet Delivery Ratio

The PDR is state that percentage of number of arriving packets by target hub to number of packets sent by all the source nodes in the stage of simulation time.

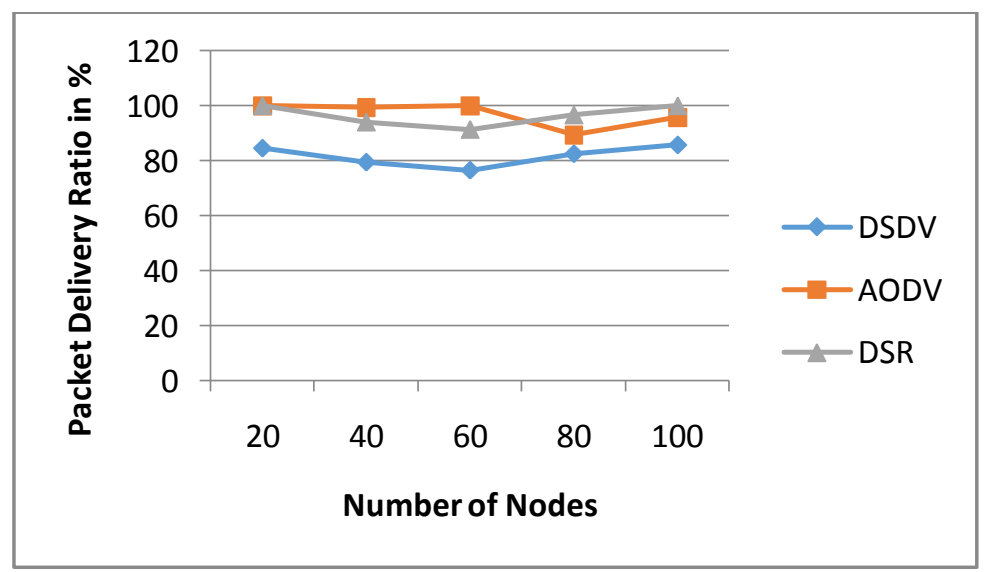

Figure 3: Packet delivery ratios 
The comparative PDR of the DSDV, AODV and DSR routing protocols are demonstrated using figure 3 with rising number of nodes. In order to symbolize the performance of routing protocols the $\mathrm{X}$ axis contains the number of nodes and the corresponding percentage of delivered packets are agreed using $\mathrm{Y}$ axis. According to the obtained results the performance of DSR routing protocol in terms of PDR (packet delivery ratio) is a lot flexible as compared to the DSDV and AODV routing protocols. Thus the performance in lessening order can be DSDV $\rightarrow$ AODV $\rightarrow$ DSR.

\section{ii. Routing Overheads}

Routing overheads is the number of control packets generated by each routing protocol throughout simulation. It is the internal calculate or efficiency any routing protocols. Two different routing protocols can use diverse amounts of overheads depending on their internal effectiveness. If control and data traffic split the same channel and channel capacity is limited then extreme control traffic often impacts data routing performance (throughput). If extra control packets are sent by the routing agents, then delay in the network will also increase.

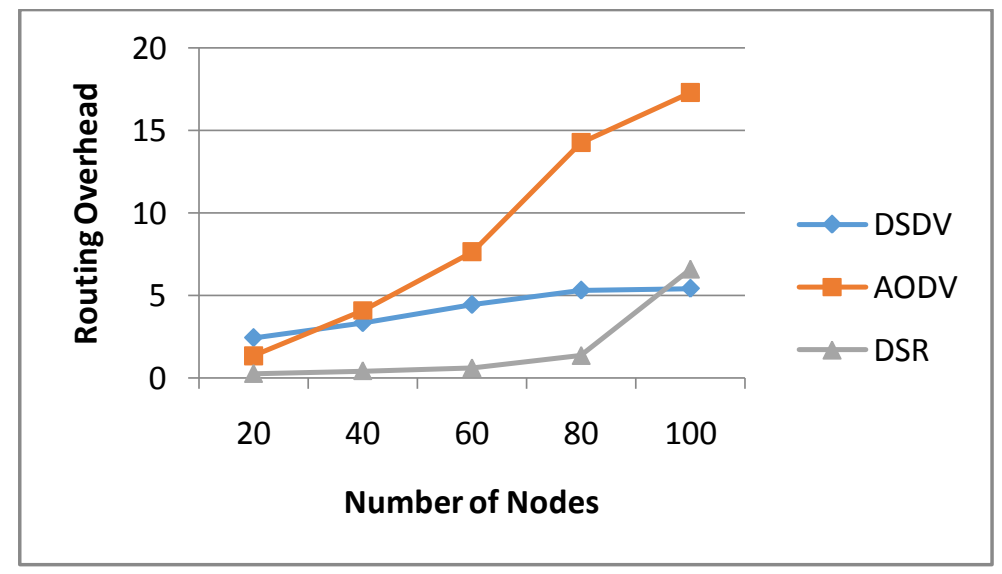

Figure 4: Routing overhead

The comparative routing overhead of the routing protocol with expanding size of movement is given figure 4. In this outline the measure of overhead is spoken to utilizing $\mathrm{Y}$ axis and the quantity of nodes are recorded in $\mathrm{X}$ axis. As per the got comes about the execution of DSR routing protocol is substantially higher than the DSDV and DSR routing as far as routing overhead. In this way DSR is more adoptable for portable specially appointed system directing.

\section{iii. Remain Energy}

The measure of preserved energy during the dynamic sessions of communication is known as remain energy. The specially appointed devices are produced with the inbuilt energy sources subsequently for every occasion in system a settled measure of energy is expended from the underlying energy. Figure 5 demonstrates the measure of remain energy in various routing sessions over expanding activity estimate.

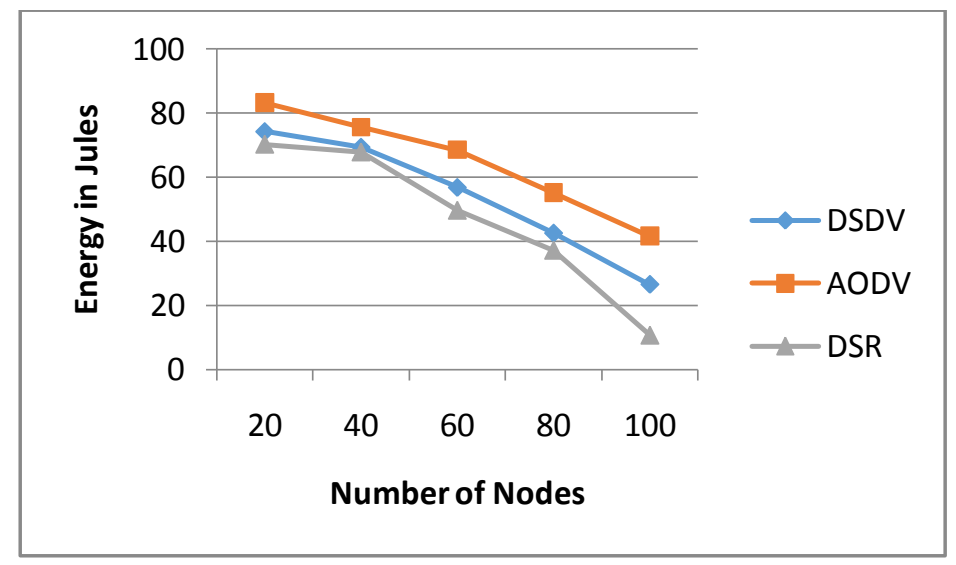

Figure 5: Energy Consumption

The amount of comparative energy expenditure is given using figure 5 in this diagram the percentage quantity of energy consumption is given in $\mathrm{Y}$ axis and the $\mathrm{X}$ axis contains the number of nodes in network. According to the evaluated results of the energy expenditure the DSR routing consumes extra energy as compared to the DSDV and 
AODV routing protocol. Notice that according to the MANET property low battery can affect the network functioning thus the DSR is not effective for the long simulations additionally the DSR is not much supportable for the large network for simulation of DSR the amount of connections are very less as compared to other two routing protocols.

\section{CONCLUSION FUTURE WORK}

This paper does the practical comparison of three routing protocols DSDV, AODV and DSR. The important surveillance is, simulation results agree with expected results based on hypothetical analysis. We find that the performance varies widely across different number of nodes and different types of speed in node mobility. According to our results, each protocol has its own applicability in different application areas. Relatively, most of the time we used AODV protocol as in many application if we compared to other two protocols. Overall, our simulation work shows that AODV performs better in a network with a large and random movement number of nodes whereas DSR and DSDV performs better when the number of nodes is less. In this paper, we have done complete analysis of the three MANET's routing protocols

In the future, wide complex simulations could be carried out using other obtainable presentation metrics, in order to increase a more in-depth performance analysis of the ad hoc routing protocols. Other new protocols performance could be studied too.

\section{REFERENCES}

[1] Stüdi, Patrick. "Quality of service for mobile ad hoc networks." (2003).

[2] Abolhasan, Mehran and Eryk Dutkiewicz, "A review of routing protocols for mobile ad hoc networks", Ad hoc networks,, pp 1-22, 2004

[3] Hong, Xiaoyan, and Mario Gerla. "Scalable routing protocols for mobile ad hoc networks." IEEE network, pp. 11-21, 2002

[4] Mäki, Silja, "Security Fundamentals in Ad Hoc Networking", Proceedings of the Helsinki University of Technology, 2000.

[5] Mishra, Amitabh, and Ketan M. Nadkarni. "Security in wireless ad hoc networks." The handbook of ad hoc wireless networks. CRC Press, Inc., 2003.

[6] Murizah Kassim et al., "Performance Analysis of Routing Protocol in WiMAX Network", IEEE International Conference on System Engineering and Technology (ICSET), 2011

[7] Cisco, Cisco Internetworking, Cisco Press, 2002.

[8] Hong, Xiaoyan and Kaixin Xu, "Scalable routing protocols for mobile ad hoc networks." IEEE network, pp. 11-21, 2002

[9] C. Perkins and E. B. Royer, "Ad hoc On-Demand Distance Vector (AODV) Routing - Internet Draft",IETF Network Working Group, July 2003.

[10] H. Ehsan and Z. A. Uzmi, "Performance Comparison of Ad Hoc Wireless Network Routing Protocols", IEEE 8th International Multi topic Conference, Proceedings of INMIC, pp.457 - 465, December 2004

[11] Haas, Zygmunt J., Marc R. Pearlman, and Prince Samar. "The zone routing protocol (ZRP) for ad hoc networks." (2002).

[12] Naserian, Mohammad, and Mohammed Tarique, "Routing overhead analysis for reactive routing protocols in wireless ad hoc networks", International Conference on Wireless and Mobile Computing, Networking and Communications, Volume 3, IEEE, 2005.

[13] Amith Khandakar, "Step by Step Procedural Comparison of DSR, AODV and DSDV Routing protocol", 4th International Conference on Computer Engineering and Technology (ICCET), Volume 40, IACSIT Press, Singapore, 2012

[14] Dilpreet Kaur and Naresh Kumar, "Comparative Analysis of AODV, OLSR, TORA, DSR and DSDV Routing Protocols in Mobile Ad-Hoc Networks", I. J. Computer Network and Information Security, pp. 39-46, 2013

[15] Rajeshkumar, V., and P. Sivakumar, "Comparative study of AODV, DSDV and DSR routing protocols in MANET using network simulator-2", International Journal of Advanced Research in Computer and Communication Engineering, (2013).

[16] Usop, and Ahmad Faisal Amri Abidin. "Performance evaluation of AODV, DSDV \& DSR routing protocol in grid environment." IJCSNS International Journal of Computer Science and Network Security, pp. 261-268, 2009

[17] Ravinder Ahuja, and Pawan Ahuja, "Performance Evaluation and Comparison of AODV and DSR Routing Protocols in MANETs under Wormhole Attack", In Proceedings of the IEEE $2^{\text {nd }}$ International Conference on Image Information Processing, ICIIP, pp. 699 - 702 , 2013

[18] Kumar, Nikhil, Vishant Kumar, and Nitin Kumar, "Comparative Study of Reactive Routing Protocols AODV and DSR for Mobile Ad hoc Networks", International Journal of Computer Science and Information Technologies, 2014.

[19] He, Guoyou. "Destination-sequenced distance vector (DSDV) protocol." Networking Laboratory, Helsinki University of Technology, pp. 1-9, 2002

[20] Ade, S. A., and P. A. Tijare. "Performance comparison of AODV, DSDV, OLSR and DSR routing protocols in mobile ad hoc networks." International journal of information technology and knowledge management, pp. 545-548, 2010 